\title{
A machine checked soundness proof for an intermediate verification language
}

\author{
Frédéric Vogels, Bart Jacobs, and Frank Piessens \\ Katholieke Universiteit Leuven, Leuven, Belgium \\ \{frederic.vogels, bart.jacobs, frank. piessens\}@cs.kuleuven. be
}

\begin{abstract}
Machine-checked proofs of properties of programming languages have gained in importance significantly over the past few years. This paper contributes to this trend by proposing an approach for doing machine-checked soundness proofs for verification condition (VC) generators. Our approach embraces the multi-phase VC generation common in modern program verifiers. Such verifiers split the generation of VCs in two (or even more) phases, using an intermediate verification language as the bridge between the programming language and logic. In our approach, we define a formal operational semantics of the intermediate verification language, and we prove the soundness of two translations separately: (1) the translation of the intermediate verification language to VCs, and (2) the translation of the source programming language to the intermediate language. This paper presents a fully machine checked proof of step (1) for a prototypical intermediate verification language, and then illustrates step (2) for a very small object oriented programming language.
\end{abstract}

\section{Introduction}

Verification condition (VC) generation is one of the classic techniques for program verification: from the program and its specification one computes a set of logical sentences (the verification conditions) whose validity implies the correctness of the program with respect to the given specification. The technique can be traced back to the very roots of program verification [1].

However, as programming languages grew more and more complex with support for features such as dynamic memory allocation, pointers, exception handling mechanisms, objects, inheritance, dynamic binding and so forth, the process of generating VCs also became significantly more complex. To master this increased complexity, many modern program verifiers $[2,3]$ split the VC generation in two phases. First the source program and its specification are compiled to an intermediate verification language, and then VCs are generated from the intermediate language. A prominent example of such an intermediate language is the BoogiePL language $[4,5]$. BoogiePL is the intermediate language of the $\mathrm{Spec}^{\sharp}$ program verifier [2] and the VCC verifying C compiler [6], and the ESC/Java line of verifiers is moving to a very similar intermediate language. 
Another unfortunate consequence of the increased complexity of VC generation for current programming languages is that soundness proofs are either omitted (and hence the VC generation is seen as some form of axiomatic semantics of the source language [4]), or are presented only informally.

This paper proposes an approach to build modular machine-checked soundness proofs for $\mathrm{VC}$ generation. We embrace the two-phase $\mathrm{VC}$ generation implemented in modern verifiers, and we structure the soundness proof in the same way. Hence, in our approach, there are two translations that need to be proven sound: (1) the translation of the intermediate verification language to $\mathrm{VCs}$, and (2) the translation of the source language to the intermediate language. This paper focuses on the first step, and gives a small example of the second step.

In Section 2, we give a short introduction to the BoogiePL ${ }^{b}$ intermediate verification language [4]. We then proceed to define its operational semantics and its VC generation algorithm. We conclude the first part with a proof of this algorithm's soundness with respect to the operational semantics. This proof is fully formalized and machine checked with the Coq proof assistant [7].

Section 3 contains a small example of step (2): we define a simple object oriented language with standard operational semantics and provide a translation to BoogiePL ${ }^{b}$, which we also prove sound.

An extended version of this paper with complete proofs is available at [8].

\section{BoogiePL $L^{b}$}

The intermediate verification language we consider is essentially the same as the language defined by Leino and Schulte [4]. We only make slight modifications to the presentation of the syntax to make the formal proofs less cumbersome. The main novelty of this section (with respect to [4]) is the definition of the operational semantics of BoogiePL $\mathrm{L}^{b}$ and the proof of the soundness of $\mathrm{VC}$ generation with respect to that operational semantics - in [4] the $\mathrm{VC}$ generation is considered an axiomatic definition of the semantics of the language.

In this section, we start off by introducing the reader to BoogiePL ${ }^{b}$ by means of an informal overview of the language (Section 2.1). Next, we formally define its syntax, operational semantics (Section 2.2) and VC generation algorithm (Section 2.3), which paves the way to a proof of BoogiePL's soundness (Section 2.4).

\subsection{Overview of the language}

A BoogiePL $L^{b}$ program consists of two parts: on the one hand, a logical part that defines constants, function symbols and axioms. The constants and functions become part of the first-order logical signature over which the VCs are formulated. The signature plus the axioms constitute a classical logical theory. As this part is fairly standard, we refer the interested reader to [4] for a more detailed description. Suffice it to say that it has an axiomatization for integers, finite maps, booleans and so forth. 
On the other hand there is the imperative part, that consists of (1) global variables which take values in the mathematical structure axiomatized by the logical part and represent (part of) the program state, and (2) a number of procedures that can be thought of as describing the possible control-flow paths in the program being verified. For example, as BoogiePL ${ }^{b}$ does not provide a heap, a translation can instead define it as a global variable being a map of (object reference, field name) pairs to values.

Procedures are parameterized operations on the state space defined by the global variables. A procedure's body consists of a single command. The following commands are provided:

- sequential composition, written $c_{1} ; c_{2}$.

- variable declaration, written var $i d$ : type which introduces a new variable with unknown initial value.

- assert expression: states that the expression must evaluate to true when execution passes that point, which can be used to specify proof obligations.

- assume expression: tells the verifier that the given expression can be assumed to be true, e.g. preconditions can be assumed to be true at the beginning of a procedure or postconditions to hold just after a procedure call.

- havoc identifier: the opposite of assume; it removes any information about the specified variable by assigning an arbitrary value to it.

- choice, written $c_{1}$ [] $c_{2}$ : represents a control flow fork: execution could continue with either $c_{1}$ or $c_{2}$. This command is typically used to model conditional branches, such as if- or while-statements.

- assignment, written $x:=$ expression: changes the variable $x$ 's value.

Since procedure specifications are so common, BoogiePL ${ }^{b}$ supports them directly: one can define procedure specifications followed by one or more procedure implementations, which all have to obey the specifications. The specifications consist of a number of requires clauses (the preconditions), ensures clauses (the postconditions), and a modifies clause, which indicates which global variables have their values changed by the procedure. Procedure definitions and calls can be treated as syntactic sugar, and hence we don't discuss them in this paper.

Details of how procedure calls can be desugared to the assertion of preconditions, a havoc on the modifies-variables followed by the assumption of postconditions can be found in [4]. We also omitted the block command which in [4] introduces a new variable scope. Blocks are not strictly necessary as it is possible to move all new local variable declaration to the method scope after having given them unique names.

\section{$2.2 \quad$ Syntax and Operational semantics}

We focus on the BoogiePL ${ }^{b}$ commands, and leave expressions abstract. We only assume that expressions define side-effect free functions from the store to the set of values.

Definition 1. The syntax is defined by the following production rules 


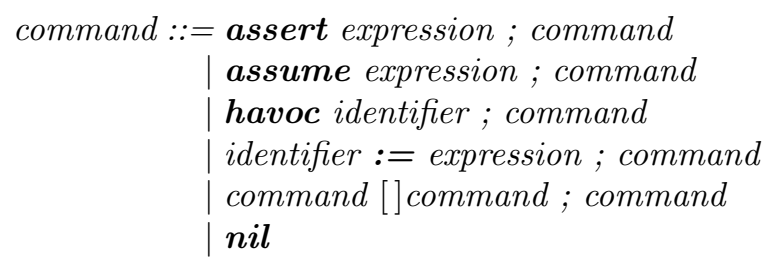

Definition 2 (Appending). We define the appending of commands, written $c_{1} \oplus c_{2}$, as

$$
\begin{aligned}
(\text { assert } e ; c) \oplus c^{\prime} & =\text { assert } e ;\left(c \oplus c^{\prime}\right) \\
(\text { assume } e ; c) \oplus c^{\prime} & =\text { assume } e ;\left(c \oplus c^{\prime}\right) \\
(\text { havoc } x ; c) \oplus c^{\prime} & =\text { havoc } x ;\left(c \oplus c^{\prime}\right) \\
(x:=e ; c) \oplus c^{\prime} & =x:=e ;\left(c \oplus c^{\prime}\right) \\
\left(c_{1}[] c_{2} ; c\right) \oplus c^{\prime} & =c_{1}[] c_{2} ;\left(c \oplus c^{\prime}\right) \\
\text { nil } \oplus c^{\prime} & =c^{\prime}
\end{aligned}
$$

We define the operational semantics as a binary relation on machine states, written $\sigma \rightsquigarrow \sigma^{\prime}$. We call this relation the single step relation as $\sigma \rightsquigarrow \sigma^{\prime}$ means that a machine in state $\sigma$ can reach another state $\sigma^{\prime}$ in one step. Note the use of the verb "can": in the context of nondeterministic semantics a state $\sigma$ can lead to (perhaps infinitely) many other states. The machine can then switch over to any one of these states. This is the case with BoogiePL ${ }^{b}$, where some commands (such as havoc and []) behave nondeterministically.

We distinguish two kinds of state:

- A failure state (fail): this state indicates that the execution went wrong;

- An in-progress state, made out of two components, written $(c \mid \mu)$

- the command $c$ : what's left of the program to execute;

- the store $\mu$ : a total mapping from identifiers to values.

Assert. The assert command verifies whether a given expression evaluates to true. If it does, all is well and execution can proceed. Otherwise, the execution goes wrong and ends up in the failure state.

$$
\begin{aligned}
& \frac{e[\mu]=\text { true }}{(\text { assert } e ; c \mid \mu) \rightsquigarrow(c \mid \mu)} \text { B-AssertTrue } \\
& \frac{e[\mu] \neq \text { true }}{(\text { assert } e ; c \mid \mu) \rightsquigarrow \text { fail }} \text { B-AssertFalse }
\end{aligned}
$$

Assume. The assume command is used to "filter out" certain execution paths. If the assume condition evaluates to true in the current store, execution can proceed as if nothing happened. Otherwise, the current execution path needn't be considered any further, which we achieve by not providing a rule for this case. Note that a state only fails if it leads to fail, hence an assume command with a false expression is not considered as failing. 


$$
\frac{e[\mu]=\text { true }}{(\text { assume } e ; c \mid \mu) \rightsquigarrow(c \mid \mu)} \text { B-Assume }
$$

Havoc. The havoc command destroys all information we have about a certain variable: havoc $x$ indicates that the rest of the program must succeed for any value of $x$. This translates to having a nondeterministic reduction rule for this command: for every possible value $v$, we start a new execution path where $x$ is bound to $v$, meaning there are infinitely many possible reductions for a state (havoc $x ; c \mid \mu$ ) because there are infinitely many values.

$$
\overline{(\text { havoc } x ; c \mid \mu) \rightsquigarrow(c \mid \mu, x \mapsto v)} \text { B-HAVOC }
$$

Assignment. An assignment updates the store with a new binding.

$$
\overline{(x:=e ; c \mid \mu) \rightsquigarrow(c \mid \mu, x \mapsto e[\mu])} \text { B-Assign }
$$

Choice. This command represents a nondeterministic choice between two execution paths: $c_{1}$ [] $c_{2} ; c$ means that both $c_{1} \oplus c$ and $c_{2} \oplus c$ need to be considered.

$$
\begin{aligned}
& \overline{\left(c_{1}[] c_{2} ; c \mid \mu\right) \rightsquigarrow\left(c_{1} \oplus c \mid \mu\right)} \text { B-ChOICELEFT } \\
& \overline{\left(c_{1}[] c_{2} ; c \mid \mu\right) \rightsquigarrow\left(c_{2} \oplus c \mid \mu\right)} \text { B-ChOICERIGHT }
\end{aligned}
$$

Definition 3 (multiple step relation).

$$
\begin{gathered}
\overline{\sigma \rightsquigarrow *} \sigma \text { B }^{*} \text {-REFLEXIVE } \\
\frac{\sigma_{1} \rightsquigarrow \sigma_{2} \sigma_{2} \rightsquigarrow * \sigma_{3}}{\sigma_{1} \rightsquigarrow^{*} \sigma_{3}} \mathrm{~B}^{*} \text {-STEP }
\end{gathered}
$$

Definition 4. We say a state $\sigma$ fails if $\sigma \rightsquigarrow^{*}$ fail, i.e. there is an execution leading from $\sigma$ to the failure state. In short, we write this as fails $\sigma$.

Definition 5. A state succeeds if it does not fail, written in short as succeeds $\sigma$.

\subsection{Weakest preconditions}

Definition 6 (Weakest preconditions).

$$
\begin{aligned}
\mathrm{wp}(\text { assert } e ; c, Q) & =e \wedge \mathrm{wp}(c, Q) \\
\mathrm{wp}(\text { assume } e ; c, Q) & =e \Rightarrow \mathrm{wp}(c, Q) \\
\mathrm{wp}(\text { havoc } x ; c, Q) & =\forall x \bullet \mathrm{wp}(c, Q) \\
\mathrm{wp}(x:=e ; c, Q) & =\mathrm{wp}(c, Q)[e / x] \\
\mathrm{wp}\left(c_{1}[] c_{2} ; c, Q\right) & =\mathrm{wp}\left(c_{1}, \mathrm{wp}(c, Q)\right) \wedge \mathrm{wp}\left(c_{2}, \mathrm{wp}(c, Q)\right) \\
\mathrm{wp}(\boldsymbol{n i l}, Q) & =Q
\end{aligned}
$$


Lemma 1.

$$
\mathrm{wp}\left(c_{1} \oplus c_{2}, Q\right)=\operatorname{wp}\left(c_{1}, \mathrm{wp}\left(c_{2}, Q\right)\right)
$$

Proof. By structural induction on $c_{1}$.

\subsection{Soundness of VC generation}

In this section we sketch the structure of the proof of the soundness theorem. A Coq development with a formalized and machine checked proof of the theorem is available at [9].

Theorem 1 (Soundness). Given a program c, its evaluation does not fail if the initial state $\mu$ fulfills the weakest precondition.

$$
\forall c, \mu \bullet \operatorname{wp}(c, \text { true })[\mu] \Rightarrow \text { succeeds }(c \mid \mu)
$$

Proof. By induction on command size. Six cases can be distinguished, two of which we discuss here. We refer to $[8,9]$ for the full details.

$-c=$ assert $e ; c^{\prime}:$ Suppose $\operatorname{wp}\left(\right.$ assert $e ; c^{\prime}$, true $)[\mu]$ is true. Then, by definition of the weakest precondition computation,

$$
\left(e \wedge \operatorname{wp}\left(c^{\prime}, \text { true }\right)\right)[\mu]
$$

is true. Hence, only B-AssertTrue applies, and we get (assert $e ; c \mid \mu) \rightsquigarrow$ $\left(c^{\prime} \mid \mu\right)$. That succeeds $\left(c^{\prime} \mid \mu\right)$ follows from the induction hypothesis and (1).

$-c=c_{1}[] c_{2} ; c^{\prime}$ : There are two possible paths for a choice command:

$$
\left(c_{1}[] c_{2} ; c^{\prime} \mid \mu\right) \rightsquigarrow\left(c_{1} \oplus c^{\prime} \mid \mu\right) \quad\left(c_{1}[] c_{2} ; c^{\prime} \mid \mu\right) \rightsquigarrow\left(c_{2} \oplus c^{\prime} \mid \mu\right)
$$

To show that both of these succeed, we need to prove that $\operatorname{wp}\left(c_{1,2} \oplus c^{\prime}\right.$, true $)$ which Lemma 1 tells us is equal to $\mathrm{wp}\left(c_{1} \oplus c^{\prime}\right.$, true $) \wedge \mathrm{wp}\left(c_{2} \oplus c^{\prime}\right.$, true $)$, which the induction hypothesis says is true.

\section{Translating to BoogiePL ${ }^{b}$}

Building a verifier for a programming language can be done by compiling the language to BoogiePL ${ }^{b}$ and then verifying the resulting BoogiePL $\mathrm{L}^{b}$ program. For the resulting verifier to be sound, the compilation of the programming language to BoogiePL $L^{b}$ needs to be correct. In this section, we describe the compilation and the correctness proof for a very small object-oriented programming language. 


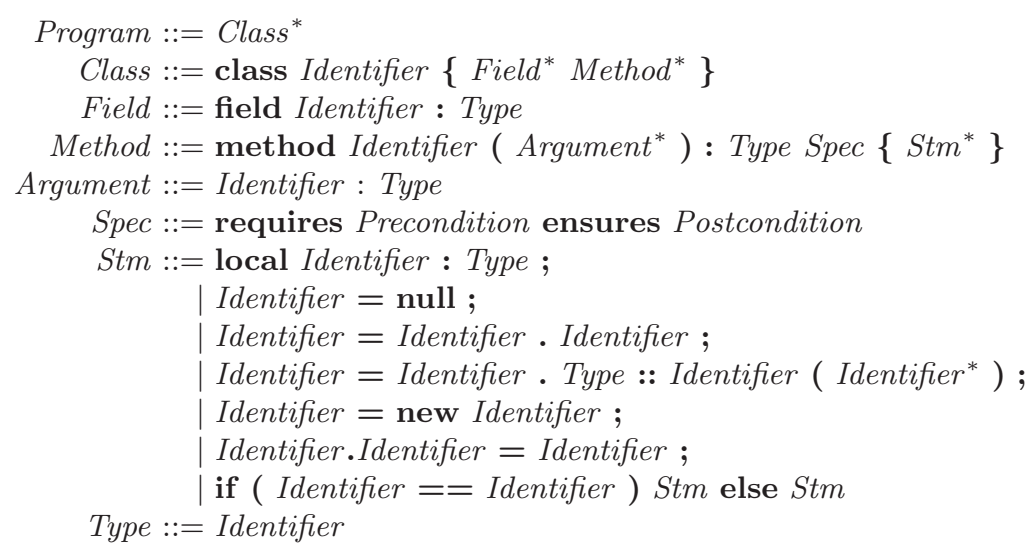

Fig. 1. Syntax of the toy OO language

\subsection{A toy OO language with contracts}

The source programming language that we start from contains both programming constructs as well as specification constructs. The syntax of the language is summarized in Figure 1. It is a very simple class-based language with pre- and postcondition declarations as specification constructs.

The semantics of the language is defined using a straightforward small-step operational semantics. The operational semantics defines all the erroneous program behaviour that the verifier is supposed to catch as going to a special FAIL state. Other program errors that are caught by other means - for instance method-not-understood errors caught by the type system - can cause the operational semantics to get stuck. For the purpose of this paper, we don't care about these errors: our soundness theorem will just say that a program that verifies will never go to the FAIL state.

The full definition of the small-step operational semantics is omitted here as it is fairly standard. Interested readers can find it in the extended version of this paper [8]. The pre- and postconditions are checked by the semantics and lead to the FAIL state if they are not satisfied. The program state is represented as a quintuplet:

- The heap: a partial function mapping object identifiers (oids) to objects, themselves partial functions mapping field names (identifiers) to oids.

- The store: a stack of frames, which are partial functions mapping identifiers to oids. It is used to store local variable bindings.

- The postcondition stack keeps track of method postconditions which need to be checked at method exit.

- The receiver stack keeps track of identifiers denoting local variables which receive the return value on method exit.

- The call stack containing statement lists; it acts as the program counter. 
Pre- and postconditions are classical logical propositions for which we do not define a syntax; instead we choose to abstract them away as functions. A method $m$ 's precondition Pre $_{m}$ needs access to its arguments and objects on the heap, which is why we model preconditions as binary functions receiving the upper frame (which contains the arguments) and the heap. The postconditions $\left(\right.$ Post $\left._{m}\right)$ can refer to the arguments, the heap state before and after the method call, and the return value. Therefore we model postconditions as functions taking an upper frame, a before-heap, an after-heap, and a return value. We'll also use partial application on postconditions.

Definition 7 (Initial state). Given a program $P$ where some class $X$ is the only to contain a nullary preconditionless T-returning method named main, the initial state $\sigma_{\text {init }}(P)$ is equal to

$$
(\epsilon, \epsilon \circ \epsilon, \epsilon, \epsilon, \bar{s} \circ \epsilon)
$$

where $\bar{s}=\operatorname{local} x: X ; \quad x=$ new $X ;$ local $r: T ; \quad r=x . X:: \operatorname{main}()$;

A verifier is supposed to check that it is not the case that $\sigma_{\text {init }}(P) \longrightarrow^{*}$ fail. We design a verifier by translation to BoogiePL $\mathrm{L}^{b}$ and we give a soundness proof of that translation.

\subsection{Modularization}

As the size of verification conditions grows quickly with the size of the BoogiePL ${ }^{b}$ program, it is essential for scalability that the verification can be done modularly. For the language under consideration, we will do verification per method: each method will be verified in isolation, using only the method body and the specifications of the methods that this method calls.

A first important part of the soundness proof is showing that this modularization is sound.

Definition 8. A program is modularly correct iff every method considered separately is correct.

Theorem 2. If a program is modularly correct, it is also correct.

We only provide a proof sketch here. The full proof is available in an accompanying technical report [8]. A key ingredient of the proof is the definition of a new modular step relation, called the step-over $(\curvearrowright)$, that executes method calls in a single step, based on their specifications. This relation is defined by the rules in Figure 2.

The following lemma states an essential connection between the operational semantics and this new step-over relation.

Lemma 2. Given a program $P$, if $\sigma_{\text {init }}(P) \longrightarrow *$ FAIL, then there exists a method $m$ from $P$ for which

$$
\exists H, F \bullet \operatorname{Pre}_{m}(H, F) \wedge\left(H, F, \operatorname{Post}_{m}(H, F), \operatorname{body}(m)\right) \curvearrowright^{*} \text { FAIL }
$$

From this lemma, Theorem 2 follows immediately. 


\begin{tabular}{|c|c|c|}
\hline H-LOCAL & $(H, F, C$, local $x: T ; \bar{s}) \curvearrowright\left(H, F^{\prime}, C, \bar{s}\right)$ & $F^{\prime}=F, x \mapsto$ null \\
\hline H-STORENULL & $(H, F, C, x=$ null; $\bar{s}) \curvearrowright\left(H, F^{\prime}, C, \bar{s}\right)$ & $F^{\prime}=F, x \mapsto$ null \\
\hline H-STOREFIELD & $(H, S, C, x=y \cdot f ; \bar{s}) \curvearrowright\left(H, S^{\prime}, C, \bar{s}\right)$ & $\begin{array}{l}\text { oid }=F[y] \neq \text { null } \\
F^{\prime}=F, x \mapsto H[\text { oid }][f]\end{array}$ \\
\hline H-STOREFIELDNULL & $(H, F, C, x=y \cdot f ; \bar{s}) \curvearrowright$ FAIL & $F[y]=$ null \\
\hline H-STORENEW & $\begin{array}{l}(H, F, C, x=\text { new } K ; \bar{s}) \\
\curvearrowright\left(H^{\prime}, F^{\prime}, C, \bar{s}\right)\end{array}$ & $\begin{array}{l}F^{\prime}=F, x \mapsto \text { oid, oid } \notin H \\
H^{\prime}=H, \text { oid } \mapsto \mathbb{F} \\
\mathbb{F}=\epsilon, \bar{f} \mapsto \text { null }\end{array}$ \\
\hline H-WRITEFIELD & $(H, S, C, x . f=y ; \bar{s}) \curvearrowright\left(H^{\prime}, S, C, \bar{s}\right)$ & $\begin{array}{l}\text { oid }=F[x] \neq \text { null } \\
\mathbb{F}^{\prime}=H[\text { oid }], f \mapsto F[y] \\
H^{\prime}=H, \text { oid } \mapsto \mathbb{F}^{\prime}\end{array}$ \\
\hline H-WRITEFIELDNULL & $(H, F, C, x . f=y ; \bar{s}) \curvearrowright$ FAIL & $F[y]=$ null \\
\hline H-IFTRUE & $\begin{array}{l}\left(H, F, C, \text { if }(x==y) s_{1} \text { else } s_{2} \bar{s}\right) \\
\curvearrowright\left(H, F, C, s_{1} \bar{s}\right)\end{array}$ & $F[x]=F[y]$ \\
\hline H-IFFALSE & $\begin{array}{l}\left(H, F, C, \text { if }(x==y) s_{1} \text { else } s_{2} \bar{s}\right) \\
\curvearrowright\left(H, F, C, s_{2} \bar{s}\right)\end{array}$ & $F[x] \neq F[y]$ \\
\hline H-CALL & $\begin{array}{l}(H, F, C, x=y \cdot T:: m(\bar{z}) \bar{s}) \\
\curvearrowright\left(H^{\prime}, F^{\prime}, C, \bar{s}\right)\end{array}$ & $\begin{array}{l}\text { oid }=F[y] \neq \text { null } \\
\operatorname{Pre}_{T:: m}(H, F) \\
\operatorname{Post}_{T:: m}(H, F)\left(H^{\prime}, S^{\prime}\right)\end{array}$ \\
\hline H-CALLPREFAIL & $(H, F, C, x=y \cdot T:: m(\bar{z}) \bar{s}) \curvearrowright$ FAIL & $\neg \operatorname{Pre}_{T:: m}(H, S)$ \\
\hline H-CALLNULL & $(H, F, C, x=y . T:: m(\bar{z}) \bar{s}) \curvearrowright$ FAIL & $F[y]=$ null \\
\hline H-EXITFAIL & $(H, F, C, \epsilon) \curvearrowright$ FAIL & $C(H, F) \neq$ true \\
\hline
\end{tabular}

Fig. 2. The step-over relation.

\subsection{Soundness of the compilation}

Compilation of the source program to BoogiePL $\mathrm{L}^{b}$ is done per method: for each method in the source program, a BoogiePL $\mathrm{L}^{b}$ program is generated according to the following compilation scheme.

The first part of the BoogiePL ${ }^{b}$ program - called the prelude and shown in Figure 3 - declares the heap as a finite map mapping (object reference, field name) pairs to values. The alloc field indicates whether an object is allocated or not. The predicates wellformed and successor express properties of heaps: in a wellformed heap fields of allocated objects point to allocated objects, and a heap is a successor of another heap if it is wellformed and contains at least the same objects. Then each method is translated according the schema shown in Figure 4 .

The compilation of statements is then straightforward. We define the compilation in full in Figure 5. A method call for instance is compiled to an assert that the receiver of the call is non-null, and that the precondition holds. Then, the heap is havoced (a sound overapproximation of the effects the method has on the heap), and then it is assumed that the new heap is a successor of the old heap (according to the successor predicate defined in the prelude) and that the postcondition of the call holds. 


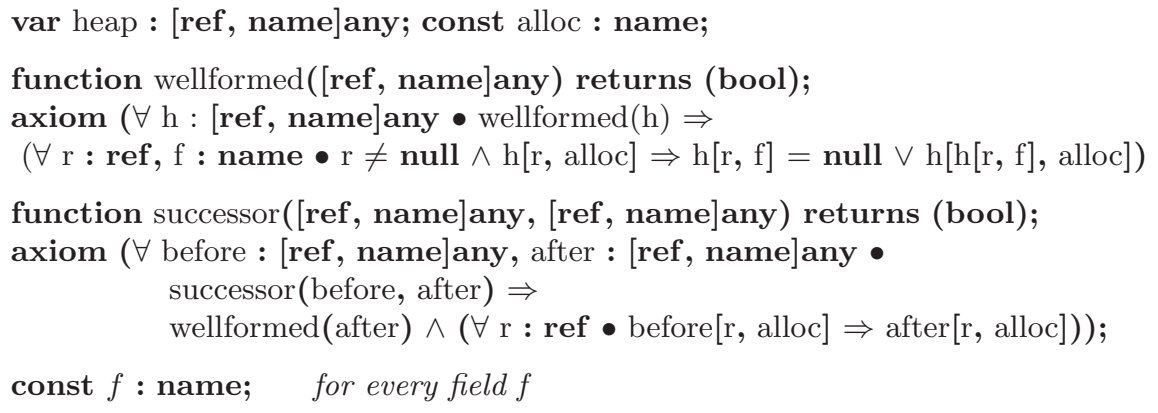

Fig. 3. Prelude of the BoogiePL $L^{b}$ program

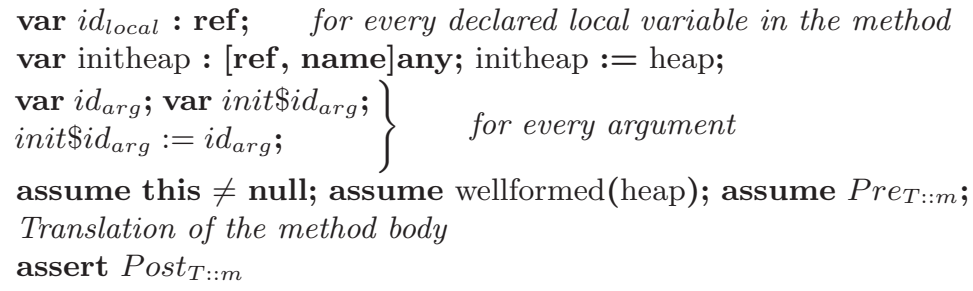

Fig. 4. Translation of a method $\mathrm{m}$

The key soundness theorem for this compilation is the following: if the method can fail under the step-over relation when started in a state satisfying its precondition, then the compiled BoogiePL ${ }^{b}$ program can also fail.

Theorem 3. Given a program $P$, let $m$ be a method of $P$ and $c$ its BoogiePL $L^{b}$ translation (see Figures 4 and 5). For all $H, F$

$$
\operatorname{Pre}_{m}(H, F) \wedge\left(H, F, \operatorname{Post}_{m}(H, F), \text { body }_{m}\right) \curvearrowright^{*} \text { FAIL } \Rightarrow \exists \mu \bullet(c \mid \mu) \rightsquigarrow{ }^{*} \text { fail }
$$

The proof consists of checking that the obvious relation between the source language program states and the BoogiePL ${ }^{b}$ program states is a simulation. We refer to [8] for the full proof.

To summarize, to ascertain that a program $P$ written in our toy language never fails we consider each method apart (modularization) and translate each into BoogiePL $\mathrm{L}^{b}$. From Theorem 3 we know that if the BoogiePL $\mathrm{L}^{b}$ translation does not fail, neither will the original method. If all methods can be shown not to fail this way, Theorem 2 guarantees that the execution of $P$ will not fail. 


\begin{tabular}{|c|c|}
\hline local $x: T$; & $\mathrm{x}:=$ null \\
\hline $\mathrm{x}=$ null; & $\mathrm{x}:=$ null \\
\hline $\mathrm{x}=\mathrm{y} \cdot \mathrm{f}$ & assert $y \neq$ null; $x:=$ heap $[y, f]$ \\
\hline $\mathrm{x}=\mathrm{y} \cdot \mathrm{m}(\overline{\mathrm{z}})$ & $\begin{array}{l}\{\text { var oldheap : [name, ref]any; } \\
\text { oldheap := heap; } \\
\text { assert y } \neq \text { null; assert Pre } e_{m} ; \\
\text { havoc heap; } \\
\left.\text { assume successor(oldheap, heap); assume } \text { Post }_{m}\right\}\end{array}$ \\
\hline $\mathrm{x}=$ new $\mathrm{T}$ & $\begin{array}{l}\{\text { var oid : ref; } \\
\quad \text { assume oid } \neq \text { null; } \\
\quad \text { assume heap[oid, alloc] }=\text { false; } \\
\quad \text { heap[oid, alloc] }:=\text { true }\}\end{array}$ \\
\hline x.f $=y$; & assert $x \neq$ null; heap $[x, f]:=y$ \\
\hline if $(\mathrm{x}==\mathrm{y}) \mathrm{s}_{1}$ else $\mathrm{s}_{2}$ & $\begin{array}{l}\left\{\text { assume } \mathrm{x}=\mathrm{y} ; \text { translation of } s_{1}\right. \\
{[]} \\
\left.\text { assume } \mathrm{x} \neq \mathrm{y} ; \text { translation of } s_{2}\right\}\end{array}$ \\
\hline
\end{tabular}

Fig. 5. Compilation scheme

\section{Related and Future Work}

There is a huge amount of related work in program verification and hence, we necessarily focus on research results most closely related to ours. For a good overview of the state-of-the-art in program verification for OO languages in general, we refer to [10].

A first closely related line of work is the Spec ${ }^{\sharp}$ verifier [2], one of the stateof-the-art automatic verifiers for Java-like languages. This paper is a first step towards a formal machine-checked soundness proof of the Spec ${ }^{\sharp}$ verifier. So far, only certain key elements of this verifier have been shown sound on paper [11].

The Why/Krakatoa/Caduceus line of tools [12] is a very interesting competitor to the Boogie/Spec $\sharp / V C C$ line of tools: both toolsets are built around a similar intermediate verification language and provide front-ends for Java-like and C-like languages. To avoid trust in the tool-chain, the Why VC generator adopts an approach where the proof produced by Why (possibly with the help of other tools or the user) is checked a posteriori by an automatic checker.

In the Mobius project [13], machine-checked proofs of many programming language properties are being studied with the purpose of supporting proofcarrying code to certify security-related properties of programs. The project has worked out a machine-checked proof of the soundness of their program logic (the Mobius Base Logic) with respect to an operational semantics of Java bytecode. As part of the Mobius project, Lehner and Müller have shown the translation of Java bytecode to BoogiePL sound [14]. This can be seen as another instance of a phase (2) soundness proof in the terminology of this paper.

This paper provides only a first step towards a machine-checked soundness proof of a Spec ${ }^{\sharp}$-like verifier. In future work, we intend to work out a phase (2) soundness proof for several variants of Spec ${ }^{\sharp}$-like verifiers. 


\section{Conclusion}

BoogiePL $\mathrm{L}^{b}$ is an intermediate language developed to assist the development of verifying compilers by simplifying the generation of verification conditions (VC) ([4]), but no formal soundness proofs existed for this language. To remedy this, this paper provides a full formalization for BoogiePL $L^{b}$ by defining its syntax, operational semantics (Section 2.2) and VC generation algorithm (Section 2.3). This paper also contains a proof of the VC generation's soundness with respect to the operational semantics; a machine-checked proof can be found at [9]. Next we have shown how soundness proofs of verifiers that translate to BoogiePL ${ }^{b}$ can be proven sound by proving the correctness of their compilation scheme.

\section{References}

1. Hoare, C.A.R.: An axiomatic basis for computer programming. Commun. ACM 12(10) (1969) 576-580

2. Barnett, M., Leino, K.R.M., Schulte, W.: The Spec\# programming system: An overview. In: Proceedings of CASSIS 04, Springer (2004) 49-69

3. Zee, K., Kuncak, V., Rinard, M.: Full functional verification of linked data structures. In: PLDI '08: Proceedings of the 2008 ACM SIGPLAN conference on Programming language design and implementation, New York, NY, USA, ACM (2008) 349-361

4. Leino, R., Schulte, W.: A verifying compiler for a multi-threaded objectoriented language. Marktoberdorf lecture notes (2007) In Manfred Broy, Johannes Grünbauer, Tony Hoare (eds.). Software System Reliability and Security. IOS Press, 2007.

5. Barnett, M., Chang, B.Y.E., DeLine, R., Jacobs, B., Leino, K.R.M.: Boogie: A modular reusable verifier for object-oriented programs. In: FMCO 2005, volume 4111 of LNCS, Springer (2006) 364-387

6. Schulte, W., Xia, S., Smans, J., Piessens, F.: A Glimpse of a Verifying C Compiler Extended Abstract (2007)

7. Bertot, Y., Castéran, P.: Interactive Theorem Proving and Program Development. Springer (2004)

8. Vogels, F., Jacobs, B., Piessens, F.: A machine checked soundness proof for an intermediate verification language: extended version. Technical Report CW526, Katholieke Universiteit Leuven (2008)

9. Vogels, F., Jacobs, B., Piessens, F.: The full Coq proof script http://www.cs.kuleuven.be $/{ }^{\sim}$ frederic/papers/boogie/boogie.v8.

10. Leavens, G.T., Leino, K.R.M., Müller, P.: Specification and verification challenges for sequential object-oriented programs. Formal Asp. Comput. 19(2) (2007) 159189

11. Barnett, M., Leino, K.R.M.: Weakest-precondition of unstructured programs. In: PASTE. (2005) 82-87

12. Filliâtre, J.C., Marché, C.: The Why/Krakatoa/Caduceus Platform for Deductive Program verification. In: CAV. (2007) 173-177

13. The Mobius Project: http://mobius.inria.fr

14. Lehner, H., Müller, P.: Formal translation of bytecode into BoogiePL. In Huisman, M., Spoto, F., eds.: Bytecode Semantics, Verification, Analysis and Transformation. Electronic Notes in Theoretical Computer Science (2007) 